\title{
Power Allocation for D2D Communications in Heterogeneous Networks
}

\author{
Yinuo He, Xi Luan, Jiajia Wang, Meiping Feng, Jianjun Wu \\ Institution of Advanced Communications, EECS, Peking University, Beijing, P.R.China \\ 100nuo@gmail.com,just@pku.edu.cn
}

\begin{abstract}
In this paper, we study power allocation for D2D communications in heterogeneous networks utilizing game theory approach to improve the performance of the whole system. Given D2D's underlay status in the system, Stackelberg game framework is well suited for the situation. In our scheme, macrocell system and femtocell system are considered as two leaders and D2D pairs are considered as the follower, forming a two-leader-one-follower Stackelberg game. The leaders act first, charging some fees from the follower for using the channel and causing interference to jeopardize their communication equality. The follower observes the leaders' behavior and develops its strategy based on the prices offered by the leaders. We analyse the procedure and obtain the Stackeberg equilibrium, which determines the optimal prices for the leaders and optimal transmit power for the follower. In the end, simulations are executed to validate the proposed allocation method, which significantly improves data rate of user equipments.
\end{abstract}

Keywords-Device-to-device, femtocell, heterogeneous networks, power allocation, LTE-A

\section{INTRODUCTION}

As a type of proximity communication, D2D communication enables user equipments (UE) to communicate with each other directly without through the evolved NodeB when UEs are in close distance [1] [2]. Therefore, D2D communication makes a great contribution in improving system throughput and extending UEs' battery lifetime. On the other hand, to provide indoor cellular users with better service quality, femtocells have gathered considerable interest recently, since they can efficiently offload traffic burden of macrocell base station (MBS), which will consequently improve network coverage and capacity [6].

Generally, as a secondary underlay which reuses the spectrum resources of the primary system, power allocation of D2D pairs is an important but challenging task to improve the performance of heterogeneous macrocell-femtocell networks [4] [5], since the receivers in this system suffer from threetier interference, i.e., macro-to-device, femto-to-device, and device-to device interference [3]. In previous SINR-thresholdbased power control method [7], only one system, either macrocell or femtocell, has been considered to determine transmit power of D2D pairs, which can be inefficient and impede sufficient deployment of D2D communications. In this paper, we study power allocation for D2D communication in heterogeneous networks utilizing game theory approach. Given D2D's underlay status in the system, Stackelberg game framework is well suited for the situation.

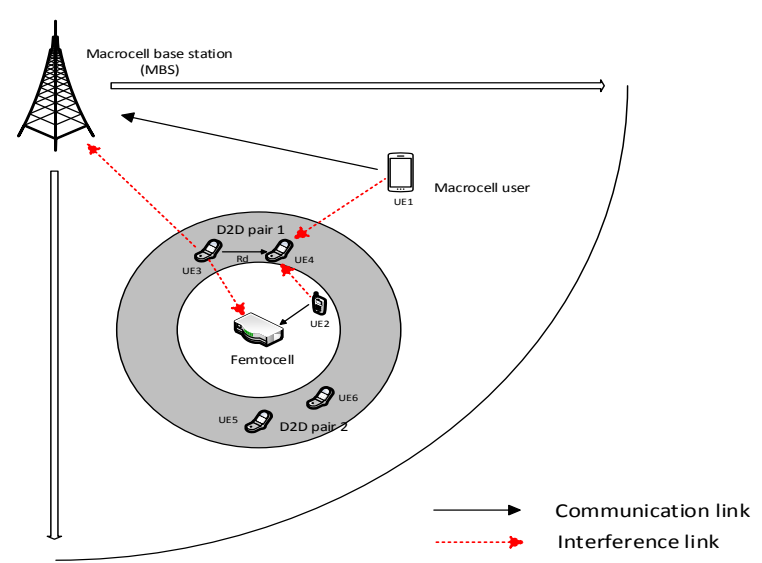

Fig. 1. System model.

The rest of this paper is organized as follows. In Section II, system model of heterogeneous networks with D2D communication as an underlay is illustrated. In Section III, we construct the Stackelberg game and formulate the equilibrium. In Section IV, simulation results are presented to validate the proposed scheme. Finally, Section V concludes the paper.

\section{System ModeL}

We consider the uplink of a Macro/Femto/D2D system in a single cell with a macrocell base station (MBS) in the center. One femtocell is randomly located in the same cell. The femtocell is assumed to be round and covers an area of $100 \pi$ square meters. The femtocell base station (FBS) is deployed at the center of the house. The femtocell serves several indoor users, which are randomly located within the house. Several macrocell UEs are distributed out of the femtocell. There are multiple outdoor D2D pairs around the considered femtocell. If D2D pairs are far enough away from the femtocell, the interference they cause to the considered femtocell can be neglected. Therefore, we locate D2D pairs around the femtocell in the shadowed area with width of $d$. The D2D UEs are in pairs, each consisting of one transmitter and one receiver between which the communication distance is $R_{d}$, as shown in Fig.1.

We assume that the number of macrocell UEs is $K$, hence there are $K$ orthogonal channels which are occupied by the corresponding macrocell UEs. The channels allocated to the macrocell UEs are fixed. The femtocell system and D2D pairs 
share channel resources with macrocell UEs. The number of the femtocell UEs is $K$. One channel is only allowed to be used by one macrocell UE, one femtocell UE and one pair of D2D UEs. In LTE, scheduling takes place every transmission time interval(TTI) [8].

\section{Stackelberg Game Formulation AND ANALYSIS}

In heterogeneous networks, interference from D2D pairs to cellular networks should be limited. Thus, transmit power of D2D UEs should be properly controlled. In this section, we first formulate this problem as a resource allocation method using Stackelberg game based scheme, then we obtain solutions of the proposed game.

\section{A. Two-level Game Framework}

Stackelberg game is employed to coordinate the scheduling. We focus on the behavior of a two-leader one-follower pair, of which a Macrocell UE and a Femtocell UE are leaders, a D2D UE is the follower. They share the same channel resource. The leaders own the channel resource and they can charge D2D UEs some fees for using the channels. The fees are fictitious money to coordinate the system. Thus, the cellular UEs have an incentive to share the channel with D2D UEs if it is profitable, and the leaders have the right to decide the prices. For D2D UEs, under the charging prices, they can choose the optimal power to maximize their payoffs.

1) D2D UEs / Follower-level: Utility of the follower can be defined as its own throughput performance minus the cost it pays for using the channel. The fees should be decided according to the leaders' consideration. Thus, we set the fee proportional to interference the leaders observe, which can be expressed as

$$
\begin{array}{r}
U_{D 2 D i}=\alpha \log _{2}\left(1+\frac{P_{i} g_{D 2 D_{i}}}{P_{1 k} g_{M_{k} D_{i}}+P_{2 k} g_{F_{k} D_{i}}+N o}\right) \\
-p_{i 1} P_{i} g_{D_{i} M_{k}}-p_{i 2} P_{i} g_{D_{i} F_{k}}
\end{array}
$$

where $P_{1 k}, P_{2 k}$ and $P_{i}$ are transmit power of the $k$-th macrocell UE, $k$-th femtocell UE and $i$-th D2D transmitter respectively. $g_{D_{i} M_{k}}$ denotes channel gain between $i$-th D2D transmitter and MBS. $g_{D_{i} F_{k}}$ denotes channel gain between the $i$-th D2D transmitter and FBS. $g_{D 2 D_{i}}$ denotes channel gain between $i$-th D2D transmitter and $i$-th D2D receiver, which are in a pair. $g_{M_{k} D_{i}}$ denotes channel gain between $k$-th macrocell $\mathrm{UE}$ and $i$-th D2D receiver. $g_{F_{k} D_{i}}$ denotes channel gain between $k$-th femtocell UE and $i$-th D2D receiver. Without loss of generality, we assume all UEs observe the same noise power $N_{0} \cdot p_{i 1}$ and $p_{i 2}$ are the charging prices $\left(p_{i 1}>0, p_{i 2}>0\right)$ of MBS and FBS respectively. We denote the set of prices for $i$-th D2D as $p_{i j}$, where $j \in\{1,2\} . \alpha$ is a scale factor to denote the ratio of the D2D's gain and its per unit of rate. $\alpha$ is a key parameter to influence the outcome of the game, which we will discuss later.

The optimization problem of follower-level game can be formulated as

$$
\max \quad U_{D 2 D i}, \quad \text { s.t. } P_{i} \geq 0
$$

2) Macrocell UEs and Femtocell UEs / Leader-level: Utility of the leaders can be defined as their gain from the follower minus the interference they observe from the D2D pair. The utility function of the leaders can be respectively described as

$$
\begin{gathered}
U_{i 1}=-P_{i} g_{D_{i} M_{k}}+p_{i 1} \beta_{1} P_{i} g_{D_{i} M_{k}} \\
U_{i 2}=-P_{i} g_{D_{i} F_{k}}+p_{i 2} \beta_{2} P_{i} g_{D_{i} F_{k}}
\end{gathered}
$$

where $U_{i 1}$ denotes utility of MBS, and $U_{i 2}$ denotes utility of FBS. $\beta$ is a factor to denote ratio of the leader's gain and the follower's payment. It mainly influence convergence speed of the proposed game.

The optimization problem of leader-level is to set a set of charging prices that maximize their utility, i.e.,

$$
\max \quad U_{i 1}, U_{i 2} \quad \text { s.t. } p_{1}>0, p_{2}>0
$$

The outcome of the proposed game will be shown in detail in the following section.

\section{B. Analysis of the Proposed Game}

In the Stackelberg game, the leaders move first and the follower moves sequentially, i.e., the leaders set the prices first, and the follower selects its best transmit power based on the price. The leaders know ex ante that the follower observes their action. Therefore, the game can be solved by backward induction.

1) Analysis of the Follower-level Game: As illustrated in (1), given $p_{i 1}$ and $p_{i 2}$ decided by the leaders, the best response of the follower is derived by solving

$$
\frac{\partial U_{D 2 D i}}{\partial P_{i}}=\frac{\alpha}{\ln 2} \frac{g_{D 2 D_{i}}}{P_{i} g_{D 2 D_{i}}+G}-p_{i 1} g_{D_{i} M_{k}}-p_{i 2} g_{D_{i} F_{k}}=0
$$

where $G=P_{1 k} g_{M_{k} D_{i}}+P_{2 k} g_{F k D i}+N o$

The solution of (6) is

$$
\hat{P}_{i}=\frac{\alpha}{\ln 2\left(p_{i 1} g_{D_{i} M_{k}}+p_{i 2} g_{D_{i} F_{k}}\right)}-\frac{G}{g_{D 2 D_{i}}}
$$

2) Analysis of the Leader-level Game: Substituting (7) into (5), we have

$$
\begin{array}{ll}
\max & U_{i 1}=\left(p_{i 1} \beta_{1}-1\right) g_{D_{i} M_{k}} P_{i}\left(\hat{p}_{i j}\right) \\
\max & U_{i 2}=\left(p_{i 2} \beta_{2}-1\right) g_{D_{i} F_{k}} P_{i}\left(\hat{p}_{i j}\right)
\end{array}
$$

We can note that (8) and (9) is a noncooperative game by MBS and FBS, and there exists a trade-off between prices and the base stations' utility. We take MBS for example. If MBS $k$ asks for a relatively low price $p_{i 1}$ at first, the D2D pair sharing the same channel will buy more power from MBS, and $U_{i 1}$ will increase as $p_{i 1}$ grows according to (8). When $p_{i 1}$ keeps growing and exceeds a certain value, it is no longer beneficial for D2D to buy so much power from MBS. In this way, $\hat{P}_{i}\left(p_{i j}\right)$ will shrink and hence results in decrement of $U_{i 1}$. Therefore, there is an optimal price for MBS to ask for. Besides, the optimal price is also affected by FBS's price. 
TABle I. Simulation Parameters and Values

\begin{tabular}{ll}
\hline Parameter & Values \\
\hline Macrocell radius & $500 \mathrm{~m}$ \\
D2D communication distance & $10 \mathrm{~m}$ \\
Shadow width (d) & $10 \mathrm{~m}$ \\
Cellular UE power & $23 \mathrm{dBm}$ \\
D2D transmit power & $0-23 \mathrm{dBm}$ \\
Thermal noise power density & $-174 \mathrm{dBm} / \mathrm{Hz}$ \\
Bandwidth & $180 \mathrm{kHz}$ \\
Transmission time interval & $1 \mathrm{~ms}$ \\
\hline
\end{tabular}

From the analysis above, by taking the derivative of $U_{i 1}$ to $p_{i 1}, U_{i 2}$ to $p_{i 2}$, and equating it to zero, we have

$$
\frac{\partial U_{i j}}{\partial p_{i j}}=\left(p_{i j} \beta_{j}-1\right) g_{i j} \frac{\partial \hat{P}_{i}}{\partial p_{i j}}+\hat{P}_{i j} g_{i j} \beta_{j}=0
$$

where $j \in 1,2$. We take $g_{i j}$ short for $g_{D_{i} M_{k}}$ when $j=1$, and for $g_{D_{i} F_{k}}$ when $j=2$.

Solving the above equations of $p_{i j}$, we denote the optimal prices as

$$
\hat{p}_{i j}=\hat{p}_{i j}\left(\left\{G_{i, j}\right\}\right)
$$

where $\left\{G_{i, j}\right\}$ denotes the set of channel gains among disparate participators.

After rearranging (10) we have

$$
p_{i j}=H_{i j}(p)=-\frac{\hat{P}_{i}}{\partial \hat{P}_{i} / \partial p_{i j}}+\frac{1}{\beta_{j}}
$$

In order to calculate $p_{i j}$ in (12), each base station listens to the instantaneous feedback information about $\hat{P}_{i}$ and $\partial \hat{P}_{i} / \partial p_{i j}$ from the D2D transmitter. Then updating of the base stations' prices can be described by a vector equality of the form

$$
\boldsymbol{p}=\boldsymbol{H}(\boldsymbol{p})
$$

where $\boldsymbol{p}=\left(p_{i 1}, p_{i 2}\right)$, and $\boldsymbol{H}(\boldsymbol{p})=\left(H_{1}(\boldsymbol{p}), H_{2}(\boldsymbol{p})\right)$, with $H_{j}(\boldsymbol{p})$ representing the price competition constraint to one base station from the other. Therefore, with the competition constraints in (13), iterations of the price updating can be expressed as:

$$
\boldsymbol{p}(t+1)=\boldsymbol{H}(\boldsymbol{p}(t))
$$

According to the price updating formula above, the leaders would obtain best prices for the follower, which will maximize their utility [9].

\section{Simulation Results}

To evaluate the performance of the proposed algorithm, we perform several simulations. We consider a single circular cell environment. The received signal power is $P_{i}=P_{j} d_{i j}^{-2}\left|h_{i j}\right|^{2}$, where $P_{i}$ and $P_{j}$ are received power and transmit power, respectively. $d_{i j}$ is the distance between the transmitter and the receiver. $h_{i j}$ represents the complex Gaussian channel coefficient that satisfies $h_{i j} \sim \mathcal{C N}(0,1)$. The scheduling takes

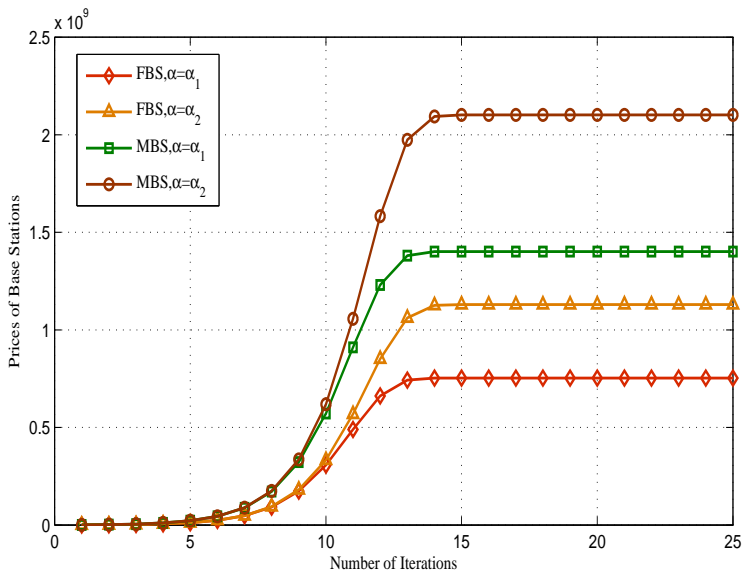

Fig. 2. Prices of MBS and FBS uder different $\alpha$

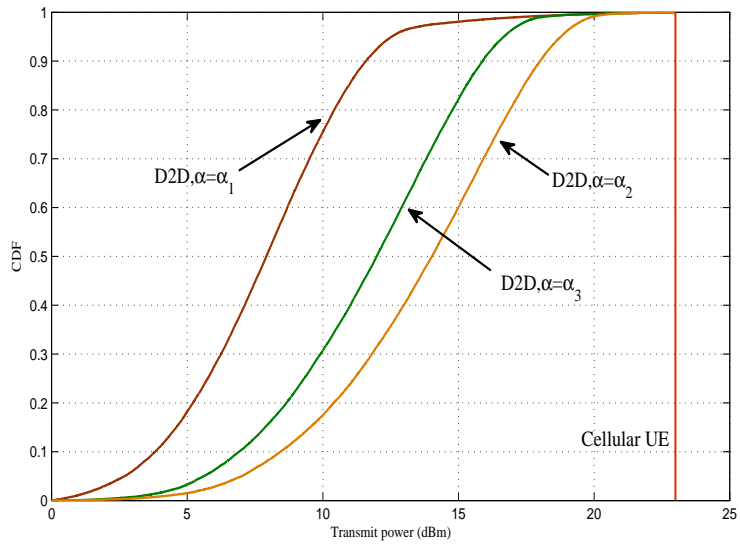

Fig. 3. Power distribution of D2D transmitter under different $\alpha$

place every TTI. Simulation parameters are summarized in TABLE I.

Simulations are conducted to observe the convergence behavior of the proposed game. In Fig.1, we plot convergence of prices for MBS and FBS under different $\alpha\left(\alpha_{1}<\alpha_{2}\right)$, where $\alpha$ denotes the gain per unit of rate as defined in (1). It is seen that the proposed scheme has fast convergence to the Stackelberg equilibrium $\hat{\boldsymbol{p}}$. It takes less than 20 iterations until the price vector $\boldsymbol{p}$ converges to the optimum. When $\alpha$ is fixed, the speed of convergence for MBS and FBS is almost the same. When $\alpha$ gets smaller, the prices asked for D2D transmission from MBS and FBS get smaller consequently. From (7), we denote that when $\alpha$ gets smaller, D2D transmitter will choose a smaller transmit power, which is illustrated in Fig.2 $\left(\alpha_{1}<\alpha_{2}<\alpha_{3}\right)$. In this case, the interference D2D causes to base stations gets relatively weaker,and there is no necessary for base stations to ask for high prices. Thus the prices are relatively lower.

In Fig.3 and Fig.4, we plot CDF of MBS and FBS rate with/without power optimization method proposed in this paper. It is clearly shown that both MBS and FBS rate is 


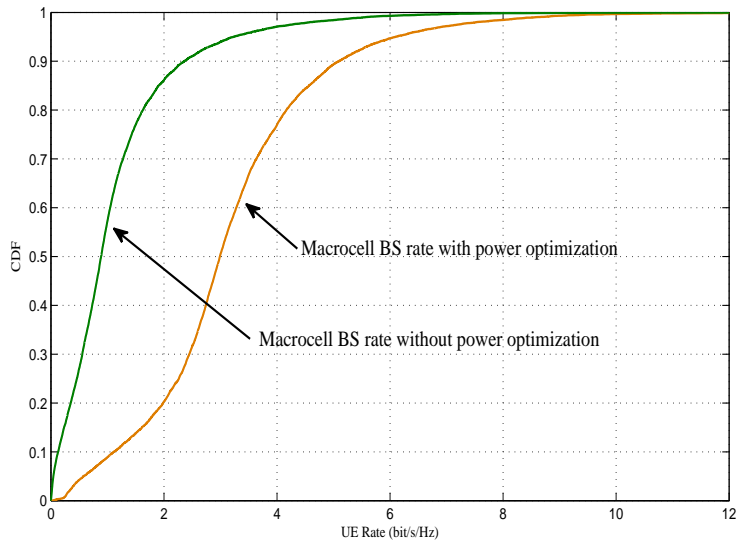

Fig. 4. Macrocell base station rate distribution

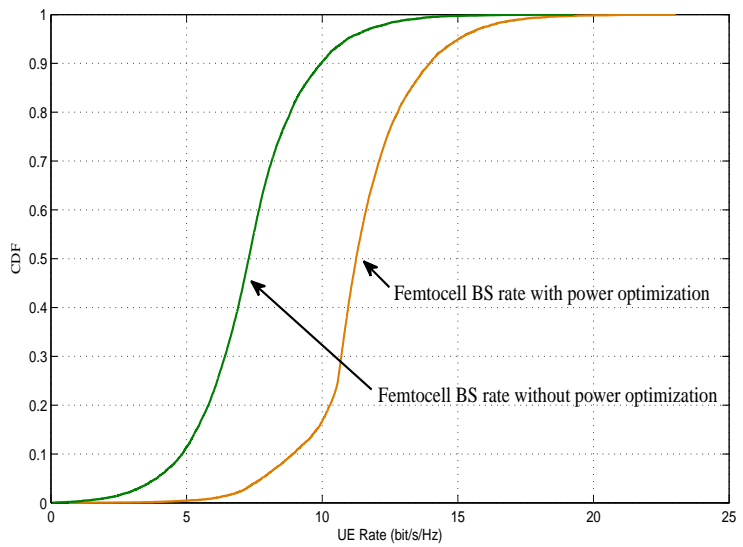

Fig. 5. Femtocell base station rate distribution

improved by approximately $2-3 \mathrm{bit} / \mathrm{s} / \mathrm{Hz}$.

\section{Conclusions}

In this paper, we constructed a Stackelberg game framework for power allocation problem of D2D communication in heterogeneous macrocell/femtocell network system. Prices and transmit power are adjusted to maximize utility obtained by base stations and D2D pairs respectively. We analyzed the optimal strategy for every participators, and worked out the solutions for equilibrium state. Simulation results have been analysed to validate the solutions, which illustrate that the proposed method can effectively promote UE rate.

\section{ACKNOWLEDGMENT}

This work is partly supported by the National Science Foundation of China (Grant No. NFSC \#61071083, \#61371073) and the National High-Tech Research and Development Program of China (863 Program), No.2012AA01A506. Corresponding author: Jianjun Wu, E-mail: just@pku.edu.cn.

\section{REFERENCES}

[1] K. Doppler, M. Rinne, C. Wijting, C. Ribeiro, and K. Hugl, Device-todevice communication as an underlay to LTE-advanced networks, IEEE Commun. Mag., vol. 47, no. 12, pp. 42C49, Dec. 2009.

[2] C.H. Yu, O. Tirkkonen, K. Doppler, and C. Ribeiro, On the performance of device-to-device underlay communication with simple power control, Proc. IEEE 69th Vehicular Technology Conference Spring, Barcelona, Apr. 2009

[3] V. Chandrasekhar, J. G. Andrews, and A. Gatherer, Femtocell networks: A survey, IEEE Communications Magazine, vol. 46, no. 9, pp. 59-67, Sept. 2008.

[4] D. Das and V. Ramaswamy, Co-channel femtocell-macrocell deployments-access control, IEEE 70th Vehicular Technology Conference Fall (VTC 2009-Fall), Anchorage, AK, Sept. 20-23, 2009. 2009

[5] P. Liu, C. Hu, T. Peng, and W. Wang, Distributed cooperative admission and power control for Device-to-Device links with QoS protection in cognitive heterogeneous network, Communications and Networking in China (CHINACOM), 2012 7th International ICST Conference, Kun Ming, Aug. 2012.

[6] C. Xu, L. Song, Z. Han, D. Li, and B. Jiao, Resource allocation using a reverse iterative combinatorial auction for device-to-device underlay cellular networks, Proc. IEEE Global Communications Conference, Anaheim, Dec. 2012.

[7] Ang-Hsun Tsai, Li-Chun Wang, Jane-Hwa Huang, and Tzu-Ming Lin, Intelligent resource management for device-to-device (D2D) communications in heterogeneous networks, Wireless Personal Multimedia Communications (WPMC), 2012 15th International Symposium, Taipei, Sept. 2012.

[8] L. Song and J. Shen, Evolved cellular network planning and optimization for UMTS and LTE. CRC Press, 2010.

[9] B. Wang, Z. Han, K.J.R. Liu, Distributed Relay Selection and Power Control for Multiuser Cooperative Communication Networks Using Stackelberg Game, Mobile Computing, IEEE Transactions on (Volume:8, Issue:7),Jul. 2009.

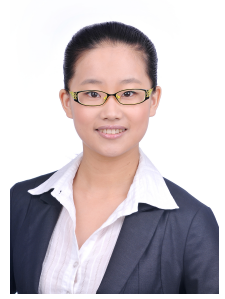

Yinuo He received her bachelor degree in electronic information science and technology from Nankai University, Tianjin, P.R. China, in 2012. Since 2012, she has been a postgraduate student in Institution of Advanced Communications, Peking University, China. Her research interests are in the area of satellite mobile communications compatible with LTE and wireless communications. Email: 100nuo@gmail.com.

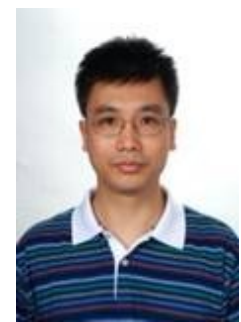

Jianjun Wu received his B.S., M.S. and Ph.D. degree from Peking University, Beijing, P. R. China, in 1989, 1992 and 2006, respectively. Since 1992, he has joined the School of Electronics Engineering and Computer Science, Peking University, and has been appointed as an associate professor since 2002. His research interests are in the areas of satellite communications, wireless communications, and communications signal processing. *The corresponding author. Email: just@pku.edu.cn. 